\title{
Setenta y cinco años de rehabilitación cardiovascular en México
}

\author{
Seventy-five years of cardiovascular rehabilitation in Mexico
}

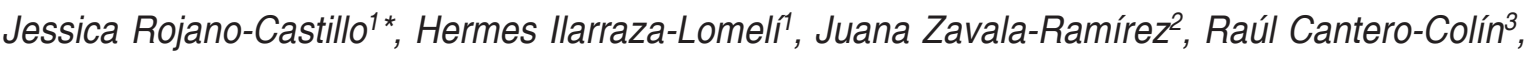 \\ Azucena Rodríguez-Reyes ${ }^{4}$, Rodolfo Lerma-Espinosa ${ }^{5}$, Marcela Mancilla-Pérez ${ }^{1}$, \\ María D. Rius-Suárez y Marianna A. García-Saldivia ${ }^{3}$
}

${ }^{1}$ Rehabilitación Cardiaca y Medicina Física, Instituto Nacional de Cardiología Ignacio Chávez; ${ }^{2}$ Rehabilitación Cardiaca, Instituto Nacional de Rehabilitación; ${ }^{3}$ Rehabilitación Cardiaca, Hospital de Cardiología, Centro Médico Nacional Siglo XXI, IMSS; ${ }^{4}$ Rehabilitación Cardiaca, Hospital General Naval de Alta Especialidad; ${ }^{5}$ Rehabilitación Cardiaca, Hospital Central Militar. Ciudad de México, México.

\begin{abstract}
Resumen
Los pacientes que sufren una enfermedad cardiovascular requieren de atención médica integral que involucre las terapias y procedimientos necesarios para reintegrarlos de forma óptima a su vida personal, familiar, laboral y social. Las intervenciones dirigidas a alcanzar dichas metas se incluyen en los programas de rehabilitación cardiaca. Estos programas son diseñados para limitar los efectos dañinos tanto fisiológicos como psicológicos de las cardiopatías, reducir el riesgo de muerte súbita o reinfarto, controlar la sintomatología cardiovascular, estabilizar o revertir el proceso de aterosclerosis y mejorar el estado psicosocial y vocacional de los pacientes. Dichos programas existen en México desde la década de 1940 y han evolucionado a lo largo de los años, adaptándose a las condiciones de enfermedad presentes en nuestro país, desde su inicio con terapias para tratar a pacientes cardiopatía reumática hasta la aplicación del ejercicio físico en pacientes con insuficiencia cardiaca, cardiopatías congénitas complejas o hipertensión arterial pulmonar. Estas actividades son de índole transdisciplinaria e implica la integración de cardiólogos, fisioterapeutas, psicólogos y nutriólogos, entre otros. Actualmente, estos programas se han extendido a lo largo de la República Mexicana gracias a cardiólogos rehabilitadores egresados de las principales instituciones de salud del país, como son los Institutos de Salud, el IMSS (Instituto Mexicano del Seguro Social) y el ISSSTE (Instituto de Seguridad y Servicios Sociales para los Trabajadores del Estado). En este documento se expondrán los orígenes de la rehabilitación, desde la época prehispánica hasta la actual, destacando las contribuciones en docencia e investigación de los médicos que han ejercido en esta área en las instituciones previamente mencionadas.
\end{abstract}

Palabras clave: Rehabilitación cardiaca. Prevención cardiovascular. México. Cardiología. Infarto. Fisioterapia.

\begin{abstract}
Patients suffering from cardiovascular disease require comprehensive medical attention that involves therapies and procedures necessary to reintegrate them optimally to their personal, family, work and social life. Interventions aimed at achieving these goals are included in cardiac rehabilitation programs. These programs are designed to limit the harmful physiological and psychological effects of heart disease, reduce the risk of sudden death or reinfarction, control cardiovascular symptoms, stabilize or reverse the atherosclerosis process and improve the psychosocial and vocational status of patients. These programs have existed in Mexico
\end{abstract}

Correspondencia:

*Jessica Rojano-Castillo

E-mail: jessy.roc@ @otmail.com
Disponible en internet: 02-10-2019 Arch Cardiol Mex. 2019;89(3):254-262 www.archivoscardiologia.com 1405-9940 @ 2019 Instituto Nacional de Cardiología Ignacio Chávez. Publicado por Permanyer México SA de CV. Este es un artículo Open Access bajo la licencia CC BY-NC-ND (http://creativecommons.org/licenses/by-nc-nd/4.0/). 
since the 1940's and have evolved over the years, adapting to the disease conditions present in our country, starting with therapies to treat patients with rheumatic heart disease until the application of physical exercise in patients with heart failure, complex congenital heart disease or pulmonary arterial hypertension. These activities are of a transdisciplinary nature and involve the integration of cardiologists, physiotherapists, psychologists, and nutritionists among others. Currently, these programs have spread throughout the Mexican Republic thanks to rehabilitation cardiologists graduating from the main health institutions in the country such as Health Institutes, Mexican Social Security Institute and Institute of Security and Social Services of State Workers. In this document, the origins of rehabilitation from the pre-Hispanic era to the present will be discussed, highlighting the contributions in teaching and research of the physicians who have practiced in this area in the aforementioned institutions.

Key words: Cardiac rehabilitation. Cardiovascular prevention. Mexico. Cardiology. Heart attack. Physiotherapy.

Quicemitqui in yollotli... (el corazón lo rige todo).

Códice Florentino.

Un paciente que egresa del hospital después de haber sufrido un evento cardiovascular continúa su proceso de convalecencia en casa, donde se recuperará integralmente. Con la intención de apoyarlo en este proceso desde hace muchos años se han desarrollado intervenciones específicamente dirigidas mediante los Programas de Rehabilitación y Prevención Cardiovascular (PRPCV). En 1964, la OMS (Organización Mundial de la Salud) publicó su primera definición operativa de este tipo de programas. Más tarde, en la década de 1980, Feigenbaum describió magistralmente a la rehabilitación cardiaca como “...programas a largo plazo que comprenden una evaluación médica, prescripción de ejercicio, modificación de factores de riesgo, educación y asesoría de los pacientes. Estos programas están diseñados para limitar los efectos dañinos tanto fisiológicos como psicológicos de las cardiopatías, reducir el riesgo de muerte súbita o reinfarto, controlar la sintomatología cardiovascular, estabilizar o revertir el proceso de aterosclerosis y mejorar el estado psicosocial y vocacional de los pacientes"'.

Estas actividades de índole transdisciplinaria buscan la reintegración del paciente a su vida cotidiana, de la manera más completa y expedita posible, con base en el entrenamiento físico, la educación y el consejo profesional. El ejercicio físico ha sido utilizado con fines medicinales, desde hace miles de años por civilizaciones como la egipcia o la griega. Hipócrates destacaba que caminar era la mejor medicina para el hombre ${ }^{2}$.En México, desde nuestros ancestros hemos reconocido al corazón como un órgano vital que requiere de estudio y cuidados. Así, el presente manuscrito busca relatar algunos de los hechos históricos notables que han hecho de la rehabilitación cardiovascular una especialidad cardiológica vigente y relevante.

«Nuestro corazón da vida, sustenta, late».

Fray Bernardino de Sahagún.
En la época prehispánica, al corazón se le consideraba como un nexo entre lo material y lo espiritual. Así, el latido constante (motlatlamotla) era sinónimo de movimiento de vida (iolli). Desde entonces se consideraba que el batir del corazón era un evento cíclico (yóllotl), lo que denominamos hoy día ciclo cardiaco. Fray Bernardino de Sahagún relataba que, para los nativos, el corazón representaba la fuente de energía y el vigor para el ser humano. Esto nos recuerda la moderna descripción que el científico sueco Per-Olof Astrand realizaría en el siglo XX, al realizar sus investigaciones sobre la utilización o consumo corporal del oxígeno $\left(\mathrm{VO}_{2}\right)^{3,4}$. Por otro lado, el códice Cruz-Badiano (Libellus de medicinalibus indorum herbis, 1552) describe síntomas cardiovasculares como la "opresión molesta del pecho, el dolor del corazón o el calor del corazón», y expone el uso de herbolaria para el tratamiento de dichas dolencias. Llama la atención la ausencia de relatos sobre algún tipo de medida preventiva ${ }^{4}$.

Aunque el entrenamiento físico no era utilizado con fines terapéuticos, el pueblo azteca apreciaba su efecto durante la preparación de sus guerreros y mensajeros (painanis), al dotarles de capacidades físicas sobresalientes ${ }^{5}$. Cabe resaltar que, aparentemente, este pueblo trataba con respeto a aquellas personas que sufrían algún tipo de deformidad o discapacidad, quienes eran incluidos socialmente ${ }^{6}$.

Una vez constituido nuestro país, en 1869 , se registra por primera vez, en el Servicio de Sanidad Militar Hospital Militar, la utilización de terapia física para pacientes con discapacidad, particularmente con el uso de baños medicinales ${ }^{7}$. Por otro lado, se tienen reportes de que un par de décadas después, en Leipzig, surgieron reportes del uso del entrenamiento físico para la atención de pacientes con enfermedad cardiovascular (Oertel, 1891)

En el mundo los siguientes años fueron marcados por revoluciones sociales como la mexicana y la rusa, así como innumerables conflictos bélicos encabezados por dos guerras mundiales. Esto condicionó 
una inevitable inestabilidad política y financiera. A continuación estudiaremos algunas de las décadas más importantes del siglo Xx y su relación con los programas de rehabilitación cardiovascular.

\section{Década de 1940}

En esta época, había en México un poco más de 6,000 médicos, y se constituyeron diversas instituciones como la Secretaría de Salubridad, los Institutos Nacionales de Salud y el Instituto Mexicano del Seguro Social (IMSS) ${ }^{9}$. Fue el 18 de abril de 1944, día en que se inauguró el Instituto Nacional de Cardiología de México (INCICh), cuando el Maestro Ignacio Chávez encomendaría la creación del área de rehabilitación cardiovascular y medicina física a su hermano, el Dr. Nicandro Chávez Sánchez, pionero de la medicina física en el país, junto con el Dr. Alfonso Tohen Zamudio (Hospital Infantil de México, 1943) y el Dr. Leopoldo Melgar Pachiano (Hospital Militar, 1946).

En ese entonces, la fiebre reumática causaba estragos en la población y exigió el desarrollo de nuevas modalidades para el diagnóstico y el tratamiento de estos pacientes, la mayor parte fundamentadas en líneas específicas de investigación. El Dr. Nicandro Chávez publicó un estudio donde mostraba el uso de la iontoforesis con salicilatos en pacientes con pancarditis reumática aguda ${ }^{10}$. Sería él quien también desarrollaría las "salas de fiebre artificial", considerando que estas acelerarían la recuperación de los pacientes. En la figura 1 se muestra al Dr. Nicandro Chávez junto a los maestros Ignacio Chávez y Enrique Cabrera.

\section{Décadas de 1950 y 1960}

Comenzaba la carrera armamentista entre los bloques capitalista y socialista, mientras la cardiopatía reumática y sus secuelas continuaban siendo un problema de salud pública. La Cardiología crecía a la par de novedosos métodos de estudio y tratamiento como las unidades de terapia intensiva y cuidados coronarios, la cirugía cardiaca con bomba de circulación extracorpórea y el primer trasplante de corazón ${ }^{11}$. México iba a la vanguardia de la Cardiología mundial en temas como fisiología, farmacología y electrocardiografía. La rehabilitación cardiovascular se mantenía sin muchos cambios $^{12}$. En la década de 1960 la cardiopatía isquémica entraba con brusquedad a la agenda cardiológica, y comenzaban a asociarse el infarto del miocardio con la aterosclerosis y la dislipidemia ${ }^{13}$. A la par, diversos investigadores destacaban la reducción de la

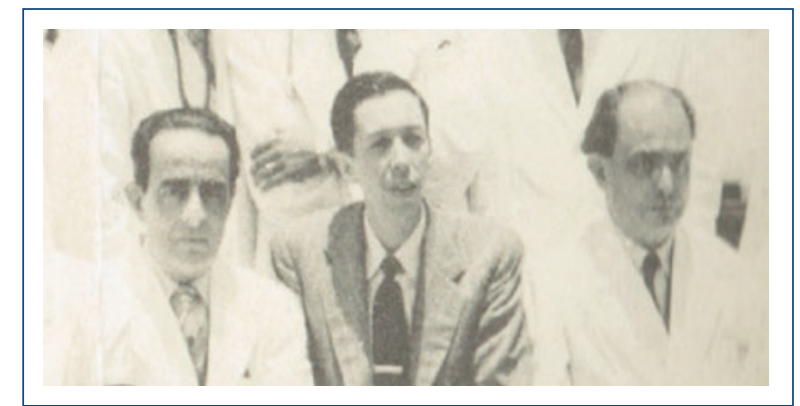

Figura 1. Fundadores de la cardiología y la rehabilitación cardiaca en México. De izquierda a derecha: Dr. Nicandro Chávez, Dr. Enrique Cabrera y Dr. Ignacio Chávez Sánchez.

mortalidad mediante la movilización temprana de los pacientes con infarto del miocardio. Por otro lado, se observó que sujetos sedentarios como los chóferes de los autobuses londinenses, tenían mayor riesgo de enfermar del corazón, en comparación con gente más activa, como los cobradores de dichos vehículos, quienes se desplazaban constantemente ${ }^{14,15}$. Fue en esta década cuando la OMS define la rehabilitación cardiaca (vide supra), hecho que atraería las miradas de múltiples investigadores interesados en la utilidad del entrenamiento físico para pacientes con enfermedad arterial coronaria ${ }^{16,17}$.

\section{Década de 1970}

Esta época estuvo marcada por el inicio de una interminable crisis financiera. En ese entonces, la Cardiología vio nacer uno de los más importantes hitos de la ciencia en el siglo Xx: la terapia de trombólisis durante el infarto agudo de miocardio. Los programas de rehabilitación cardiaca se enfocaban al manejo de los pacientes encamados, buscando la movilización temprana, debida principalmente a la prolongada estancia hospitalaria, y naturalmente surgía el interés específico en el manejo de pacientes con enfermedad coronaria ${ }^{18,19}$.

En esta era se incorporaron nuevos actores, como Juan Enrique Quintal Velazco, médico especialista en rehabilitación, quien tuvo una breve estancia en el Instituto Nacional de Cardiología para continuar como jefe de Servicio en nosocomios como el Hospital General de México, el Hospital López Mateos y el Centro Hospitalario 20 de noviembre del Instituto de Seguridad y Servicios Sociales de los Trabajadores del Estado (ISSSTE). En esta última institución se fundó el Servicio de Rehabilitación Cardiovascular en 197420,21.

Paralelamente, el 1 de agosto de 1975, el cardiólogo Jorge Escudero de la Peña daría comienzo a 
las actividades del programa de rehabilitación cardiovascular en el IMSS, dentro de las instalaciones de lo que fuera el Hospital de Enfermedades del Tórax, hoy Hospital de Cardiología del Centro Médico Nacional Siglo XXI (CMNsXXI). A principios de esta década, Nicandro Chávez se jubilaría del INCICh y sería sustituido por Pedro Fernández de la Vega y posteriormente Jorge Marcushamer. Los programas de rehabilitación cardiovascular fueron adquiriendo una estructura multidisciplinaria que integraba el trabajo de médicos, enfermeras y fisioterapeutas en tres fases: intrahospitalaria (I), hospitalaria con pacientes externos (II) y domiciliaria (III) 22 .

La investigación en los centros mexicanos de rehabilitación cardiovascular se centró en la movilización temprana $(\mathrm{FI})$, el entrenamiento físico en sujetos con cardiopatía isquémica, insuficiencia cardiaca, posoperados de cirugía de revascularización y posintervencionismo coronario, así como la prevención primaria y secundaria. A la par, se desarrollaron protocolos sobre la utilización de la prueba de ejercicio en diferentes tipos de pacientes y se publicaron trabajos sobre la adaptación de algunos deportes para el entrenamiento de pacientes en rehabilitación cardiovascular (vóleibol), la instauración de clínicas para el abandono del tabaquismo, el papel de la psicología y el trabajo social en estos programas, y el efecto de la rehabilitación cardiovascular en el retorno laboral. En la figura 2 se muestran las publicaciones realizadas durante estos 75 años de historia, clasificadas por institución y tipo de publicación.

\section{Década de 1980}

Estos años estuvieron representados por el final de la guerra fría, la caída del muro de Berlín y la desmantelación de la Unión Soviética a consecuencia de la apertura de Mijail Gorbachov (Perestroika). Así también, se implantaron los primeros cardiodesfibriladores y realizaron las primeras angioplastias ${ }^{23}$.

La rehabilitación cardiovascular se integró formalmente a los programas de prevención cardiovascular, consolidando robustos programas de entrenamiento físico y educación, enfocados primordialmente a pacientes con cardiopatía isquémica. En la Sociedad Europea de Cardiología, el grupo de trabajo encabezado por los países escandinavos, Alemania, Suiza y España dominaban el panorama en el viejo continente mientras que en EE.UU., Froelicher y Humprey eran los líderes ${ }^{24,25}$.

En el INCICh, diversos personajes laboraron en el Servicio de Rehabilitación Cardiovascular, destacando

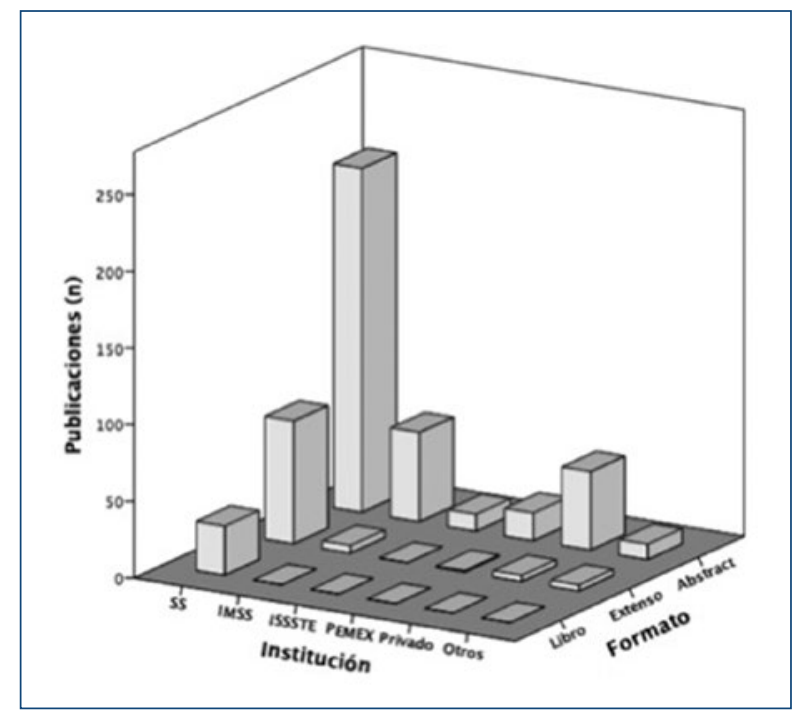

Figura 2. Investigación en rehabilitación cardiaca. Publicaciones por institución y tipo: libros, artículos en extenso o trabajos libres en congreso. SS: Servicios de Salud (incluye Instituto Nacional de Cardiología Ignacio Chávez, Instituto Nacional de Rehabilitación, Instituto Nacional de Ciencias Médicas y Nutrición Salvador Zubirán, Hospital General de México e Instituto Nacional de Pediatría); IMSS: Instituto Mexicano del Seguro Social; ISSSTE: Instituto de Seguridad y Servicios Sociales para los Trabajadores del Estado.

entre otros Jorge Marcushamer, Javier Marín, Luis Méndez, Jesús A. González Hermosillo, Ma. Guadalupe Hernández, Jorge Hernández, Luis Villamar y Juana Pineda, Marcela Pérez y Sofía Bouchet. En esta época se realizaron las primeras pruebas cardiopulmonares de ejercicio con el uso de la bolsa de Douglas. Los programas de docencia especializada en rehabilitación cardiovascular dieron inicio, tanto para cardiólogos, como para enfermeras y fisioterapeutas ${ }^{26}$.

En el CMNsXXI-IMSS, Edmundo Pombo sustituiría a Juan Rivera en la jefatura del Servicio de Rehabilitación Cardiovascular, fechas en las que se incorporaron el también cardiólogo Héctor Hernández y Hernández, la enfermera Socorro Olvera, el técnico en electrodiagnóstico Roberto González y las fisioterapeutas Margarita Marín, Amira Morales y Patricia Rosas. En el año 1985, el Dr. Hernández sustituiría al Dr. Pombo en la jefatura del Servicio.

\section{Década de 1990}

Es el nacimiento de la Unión Europea y la novedad en comunicaciones que se llamó Internet. Los procedimientos intervencionistas estaban ya en boga y la 
prevención cardiovascular adquiría cada vez mayor presencia ${ }^{27}$. Varios Servicios de Rehabilitación Cardiovascular surgieron en diversas instituciones públicas y privadas de nuestro país.

El primer antecedente de un censo de centros de rehabilitación cardiaca en México fue realizado en 1993 y presentado en el Congreso Nacional de Cardiología en la ciudad de Veracruz. Los resultados se presentaron en un cartel que identificaba a ocho centros especializados en rehabilitación cardiovascular, donde se ofrecían programas de entrenamiento físico (37\%), educación al paciente y familia (62\%), control de estrés $(50 \%)$, nutrición $(37 \%)$, clínica antitabaco $(12 \%)$, consejo laboral $(25 \%)$ y consejo sobre actividad sexual $(50 \%)^{28}$.

Gabriel Vázquez-Cabrer dirigía el programa de rehabilitación cardiovascular en el INCICh, e implementó, junto con la enfermera Guadalupe Pinson, un programa basado en su experiencia recabada de unidades en España, Alemania y EE.UU. En su época, se estudió el efecto del entrenamiento físico en pacientes con insuficiencia cardiaca, posrevascularizados, la reintegración laboral de los enfermos y la calidad de vida de los pacientes. A finales de la década, Bonifacio Mendoza sustituiría a Gabriel Vázquez. En el INCICh recibieron formación académica sobre rehabilitación cardiovascular diversos de médicos, fisioterapeutas y enfermeras del país y del extranjero.

En el CMNsXXI-IMSS, el Servicio estaba conformado por los cardiólogos Héctor Hernández, Patricia Camacho y Noemí Contreras, y el fisioterapeuta René Cámara, entre otros. Ahí se atendían pacientes con enfermedad valvular, cardiopatías congénitas, hipertensión arterial sistémica e intervencionismo coronario. Esa década fue de desarrollo, tanto en el área física como en la incorporación de personal en medicina física, psiquiatría, endocrinología, fisioterapia, electrodiagnóstico, enfermería, trabajo social y nutriología. En el año 1991 se creó el capítulo de Fisioterapeutas en Rehabilitación Cardiaca de la Asociación Nacional de Cardiólogos del IMSS (ANCIMSS), actualmente la Asociación Nacional de Cardiólogos de México (ANCAM).

En el año de 1992, Gabriel Cherebetiu, médico rumano, fue pionero de la especialidad de medicina del deporte en nuestro país y fundó el Servicio de Rehabilitación Cardiovascular en el Hospital Ángeles del Pedregal, como parte del Departamento de Fisiología Cardiovascular, mismo que dirige desde entonces. En el año 1994 se constituye la Asociación Mexicana de Rehabilitación Cardiovascular y Pulmonar A.C, primera en su género en nuestro país.
El cardiólogo Manuel Antonio Leyva Gómez inició en 1995 la Unidad de Rehabilitación Cardiovascular del Hospital Central Sur de los servicios médicos de Petróleos Mexicanos (PEMEX) y participó activamente en diversas actividades académicas junto con otras instituciones.

Ese mismo año dio comienzo el programa de rehabilitación cardiovascular en el Instituto Nacional de Rehabilitación (INR), de la mano de la médico en rehabilitación Juana Zavala Ramírez, el cardiólogo Luis Jesús San Juan Rivas, Víctor Suárez, la psicóloga Angélica Ayala Balcázar, y las terapeutas Mónica Garza Hevia, Maira Mar Fadanelli, Graciela Valero, Carmen Rosales y Martha Ríos. Carmen Gutiérrez de Velasco era entonces la jefa de la División de Rehabilitación Geriátrica y Cardiorrespiratoria. Un par de años después, en el Centro Médico Naval fue creado el Servicio de Rehabilitación Cardiovascular por el cardiólogo Héctor Sánchez Sierra junto con la enfermera Hugolina Márquez de La Paz (1 de julio de 1997).

A finales de la década de 1990, Macías Hidalgo publicaría diversos trabajos sobre el programa de rehabilitación cardiovascular que llevaba en el Hospital de Enfermedades Cardiovasculares y del Tórax CMNN IMSS en la ciudad de Monterrey, NL, siendo los principales temas de investigación el entrenamiento físico en pacientes con cardiopatía y su efecto en la calidad de vida, la adherencia a los programas de rehabilitación cardiovascular y la detección temprana de enfermedad coronaria en la mujer.

\section{Del año 2000 al presente}

La llegada del nuevo milenio estuvo acompañada por la crisis económica mundial, la guerra contra el terrorismo y la creación de los teléfonos inteligentes, que han revolucionado las comunicaciones a nivel personal. En la República Mexicana se produce una transición política inédita y toma posesión Vicente Fox como el presidente de oposición de la República Mexicana.

En el Instituto Nacional de Cardiología, Hermes llarraza Lomelí llegó a la jefatura del Servicio y adaptó un programa de rehabilitación y prevención cardiovascular basado en la escuela europea de países como Suiza, España, Alemania, Suecia e Italia. Dicho programa transdisciplinario incluye las tres áreas del quehacer médico: asistencia, docencia e investigación. A las líneas de investigación previamente establecidas se incorporaron el estudio de la prueba de ejercicio cardiopulmonar, calculadoras de estratificación de riesgo, el entrenamiento físico en niños con cardiopatía y 


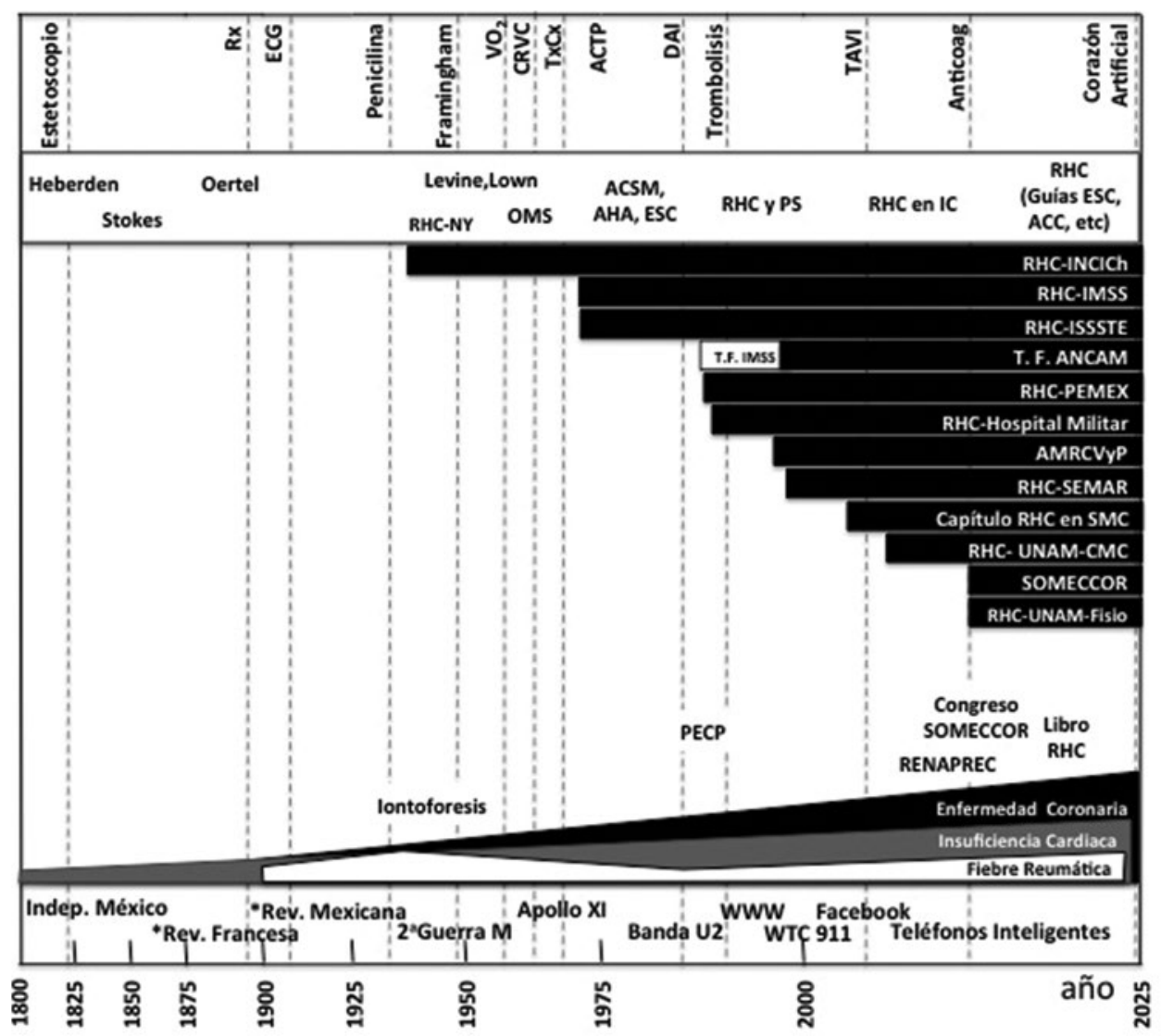

Figura 3. Cronología de la RHC en la historia de la cardiología, la historia mexicana y la universal. Eje horizontal: en la parte inferior: grupos de 25 años, iniciando en 1800 y proyectándose al 2025. En el mismo eje horizontal, en la línea inmediata superior a los años: algunos de los eventos importantes de la historia universal y de México. Parte superior de la figura: algunos eventos que cambiaron la historia de la medicina y la cardiología (invención de estetoscopio, Rx, ECG, entre otros); En la barra blanca horizontal superior, extremo izquierdo: personajes famosos y eventos de la historia de la RHC mundial, iniciando con Sir William Heberden; Extremo derecho: Guías de RHC de las principales sociedades de cardiología; Centro de figura: instituciones que cuentan con servicios de RHC; Extremo izquierdo de la barra: año de inauguración del servicio de cada institución, así como los años de fundación del capítulo de RHC en la Sociedad Mexicana de Cardiología, el reconocimiento por el Consejo Mexicano de Cardiología, la fundación de la Sociedad Mexicana para el Cuidado del Corazón (SOMECCOR) y el reconocimiento de la Universidad Nacional Autónoma de México (UNAM) para los cursos de alta especialidad de RHC para cardiólogos y el Diplomado de fisioterapia en RHC; Inferior a las barras de las instituciones: eventos clave en RHC, como la iontoforesis, el inicio en el uso de la prueba de esfuerzo cardiopulmonar, el primer Registro Nacional de Programas de RHC en México, el primer Congreso Mexicano de RHC, Prevención y Cardiología del Deporte y los libros acerca de RHC; Inferior a los eventos de la RHC en México, señaladas en blanco, gris y negro: las principales patologías que se investigan en cardiología según publicaciones de PubMed. Rx: rayos X; ECG: electrocardiograma; V02: consumo de oxígeno; CRVC: cirugía de revascularización coronaria; TxCx: trasplante cardiaco; ACTP: primera angioplastia; DAl: primer desfibrilador; TAVI: transcatheter aortic valve replacement, RHC-NY: rehabilitación cardiaca en Nueva York; ACSM: American College of Sports Medicine; AHA: American Heart Association; ESC: European Society of Cardiology, RHCyPS: rehabilitación cardiaca y prevención secundaria; RHC en IC: Rehabilitación cardiaca en insuficiencia cardiaca; ACC: American College of Cardiology, INCICh: Instituto Nacional de Cardiología Ignacio Chávez; IMSS: Instituto Mexicano del Seguro Social; ISSSTE: Instituto de Seguridad y Servicios Sociales para los Trabajadores del Estado; TF: terapia física; ANCAM: Asociación Nacional de Cardiólogos de México; PEMEX: Petróleos Mexicanos; AMRCVyP: Asociación Mexicana de Rehabilitación Cardiovascular y Pulmonar; SEMAR: Secretaría de Marina; SMC: Sociedad Mexicana de Cardiología; CMC: Consejo Mexicano de Cardiología; UNAM: Universidad Nacional Autónoma de México; SOMECCOR: Sociedad Mexicana para el Cuidado del Corazón; PECP: prueba de esfuerzo cardiopulmonar; RENAPREC: Registro Nacional de Programas de Rehabilitación Cardiaca. 
pacientes de muy alto riesgo, aplicación de las ciencias de la complejidad, la percepción del esfuerzo y la adaptación de nuevas modalidades de actividad física como el baile de salón o el tenis modificado.

Los programas universitarios de alta especialidad de Rehabilitación Cardiaca enfocados para la formación de médicos, incluyeron primero a especialistas en medicina de rehabilitación (INR-2006) y posteriormente a cardiólogos (UNAM, 2007), habiéndose graduado a la fecha decenas de residentes. De manera análoga, el curso de formación de fisioterapeutas en rehabilitación cardiovascular está avalado por la UNAM desde el 2014.

En el CMNsXXI-IMSS se cuenta con ambos programas. Estos cursos, con modalidad teoricopráctica y una adecuada carga de labor asistencial, constituyen modelos mundiales únicos en la formación de este tipo de recursos humanos. En el año 2012, el Consejo Mexicano de Cardiología otorgó el certificado de idoneidad a los programas de rehabilitación cardiaca y prevención secundaria como adiestramiento de Alta Especialidad en Cardiología. En el CMNsXXI-IMSS, Rodolfo Herrera tomó la jefatura del Servicio e incorporó la rehabilitación cardiovascular al programa de trasplante cardiaco. Otros médicos que destacaron en el IMSS y que impulsaron el desarrollo de la rehabilitación cardiaca en dicha Institución fueron Samuel Justiniano, Raúl Cantero, Jorge Hilario Jiménez y Daniel Gasca.

En los servicios médicos de la Armada de México, José Luis Álvarez Cabrera continuaría la labor de Héctor Sánchez Sierra como jefe de Rehabilitación Cardiovascular, junto con Carlos Buenfil. Al inaugurarse las nuevas instalaciones del Hospital General Naval de Alta Especialidad (HOSGENAES) en 2008, la dirección del Servicio quedaría en manos del cardiólogo Joel Álvarez Peña, quien sería sustituido posteriormente por Azucena Rodríguez ${ }^{29}$.

En el año 2006 ingresa como jefe de División al Instituto Nacional de Rehabilitación el médico rehabilitador Álvaro Lomelí Rivas, quien logra que se inicie la Alta especialidad en Rehabilitación Cardiaca y Juana Zavala participaría como profesora adjunta. El INR se especializa en la atención de pacientes con enfermedad cardiovascular y diferentes tipos de discapacidad, particularmente pacientes con amputaciones de miembros inferiores. Posteriormente se integra a este equipo el cardiólogo Francisco Ramos. Unos años después, la jefatura pasaría a manos de Juana Zavala, quien fuera sustituida por Ángela Solache Carranco y posteriormente por Roberto Carlos Sahagún Olmos.
En marzo de 2003 se fundó el capítulo de Rehabilitación Cardiaca en la Sociedad Mexicana de Cardiología, mismo que ha tenido una activa participación tanto en sesiones estatutarias como en los congresos nacionales.

En mayo de 2013 se constituyó la Sociedad Mexicana para el Cuidado del Corazón (SOMECCOR) con la misión de llevar a cabo el estudio, la investigación, la docencia, el ejercicio, la divulgación y la aplicación de los conocimientos de la rehabilitación cardiovascular, cardiología del deporte y ciencias afines. A lo largo de estos años se han auspiciado más de 60 sesiones académicas ordinarias y se llevaron a cabo dos congresos nacionales (León 2013 y Veracruz 2016). La SOMECCOR es reconocida por el Consejo Mexicano de Cardiología como una asociación cardiológica dedicada a la actualización de especialistas y está integrada por siete capítulos: Cardiología, Medicina de rehabilitación, Medicina del deporte, Enfermería, Fisioterapia, Prevención y Nutrición-Psicología ${ }^{30}$.

Es importante remarcar la participación de los pacientes en la constitución de dos sociedades llamadas Enfermos del Corazón A.C. y Cardiópatas Unidos A.C., con asociados tanto del CMNsXXI como del Instituto Nacional de Cardiología. En la actualidad existen diversas redes sociales que brindan apoyo a este tipo de pacientes.

A la fecha, el Instituto Nacional de Cardiología y el CMNsXXI han incrementado su base laboral con la incorporación de Aurora Palao y Jesús Silva en el IMSS $^{31}$ y Jessica Rojano Castillo en el INCICh. En la figura 3 se resumen los eventos más importantes en la historia universal, Cardiología y rehabilitación cardiaca. En la figura 4 se incluyen algunos de los médicos, fisioterapeutas y enfermeras que han contribuido al desarrollo de la rehabilitación cardiaca en México.

En resumen, a lo largo de este artículo se ha mostrado cómo los diferentes programas de rehabilitación cardiaca han surgido y evolucionado en nuestro país desde 1944, como una continua adaptación a los requerimientos de cada época y se han consolidado como una parte fundamental en el tratamiento integral en la actualidad. El futuro encierra muchos retos, como la consolidación de los programas de rehabilitación cardiovascular para pacientes con cardiopatías congénitas o con hipertensión arterial pulmonar, además del grave problema que conlleva la baja tasa de referencia de pacientes elegibles para programas de rehabilitación cardiovascular, junto con la pobre adherencia de los pacientes al tratamiento médico. Una de las claves para hacer frente a estos retos se basa en el espíritu 


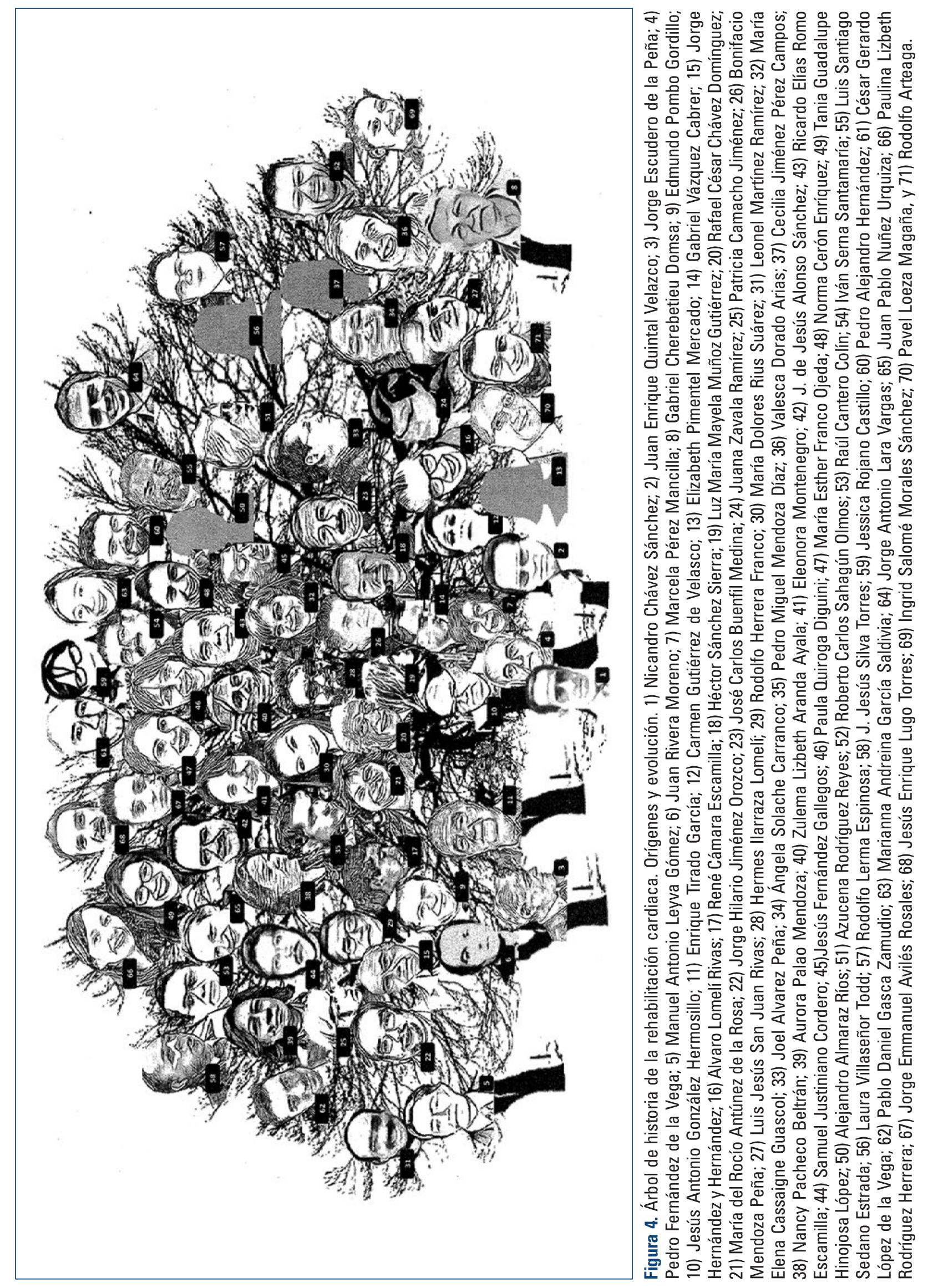


de trabajo en equipo, transdisciplinario y enfocado a la resolución de los problemas inherentes a los pacientes con cardiopatía.

«Necesitamos crear nosotros mismos, hacer ciencia nosotros mismos y no pasarnos la vida rezando las verdades y los errores que nos legaron otros. Mientras no hagamos eso, seremos los eternos ignorados en el mundo científico y los eternos incapaces para resolver nuestros problemas de acuerdo con la realidad propia».

M. Ignacio Chávez

\section{Agradecimientos}

A la Dra. Liliana Lucatero Lecona, del Centro Médico Nacional 20 de noviembre, ISSSTE (Ciudad de México).

Agradecemos la colaboración de todos y cada uno de los integrantes de las diversas unidades que han permitido a los programas de rehabilitación cardiaca cumplir 75 años de existencia en nuestro país.

\section{Conflicto de intereses}

Los autores declaran no tener conflicto de intereses.

\section{Financiamiento}

La presente investigación no ha recibido ayudas específicas provenientes de agencias del sector público, sector comercial o entidades sin ánimo de lucro.

\section{Responsabilidades éticas}

Protección de personas y animales. Los autores declaran que para esta investigación no se han realizado experimentos en seres humanos ni en animales.

Confidencialidad de los datos. Los autores declaran que en este artículo no aparecen datos de pacientes.

Derecho a la privacidad y consentimiento informado. Los autores declaran que en este artículo no aparecen datos de pacientes.

\section{Bibliografía}

1. Feigenbaum E, Carter E. Cardiac rehabilitation services. Health technology assessment report, 1987, no. 6. Rockville (MD): US Department of Health and Human Services, Public Health Service, National Center for
Health Services Research and Health Care Technology Assessment. DHHS publication no. PHS 88-3427, 1988.

2. Toscano W. Los ejercicios físicos y la salud en el corpus hipocrático Consideraciones a tener en cuenta para la educación física [Internet]. [Consultado el 30 de junio de 2018]. Disponible en: http://www.cienciared.com.ar/ra/usr/41/626/calidadevidauflo_i_pp67_82.pdf

3. Carlos Viesca T, Aranda C, Ramos de Viesca M. El corazón y sus enfermedades en la cultura náhuatl prehispánica. Estudios de Cultura Náhuatl. 2005;36:225-43.

4. Laín Entralgo P. Historia de la medicina. Barcelona, España: Salvat;1978.

5. Painani, los mensajeros del imperio azteca. Consultado el 11 de junio de 2019. Disponible en: http://www.runthehistory.com/2017/09/painani-los-mensajeros-del-imperio-azteca.html

6. Ruiz L, Devesa I. La medicina de rehabilitación en la segunda mitad del siglo XX. México, Univerdidad Nacional Autónoma de México; 2005.

7. Lerma R. Historia de la rehabilitación en Fuerzas Armadas. Sociedad Mexicana para el Cuidado del Corazón A.C. Rehabilitación Cardiovascular, Prevención y Cardiología del Deporte. Comunicación personal, 2018.

8. Oertel MJ. Allgemeine Therapie der Kreislauf-Störungen, Kraftabnahme des Herzmuskels, Ungenügende Compensation bei Herzfehlern. Leipzig: F.C.W. Vogel; 1891.

9. Flisser A. La medicina en México hacia el siglo XX. Gac Méd Méx. 2009;145:353-6.

10. Guerra F, Chávez N. El valor de la lontoforesis con salicilato de sodio en el reumatismo cardiovascular. Archivos del Instituto de Cardiología de México. 1945;15(XV):153-60.

11. Mehta N, Khan I. Cardiology's 10 Greatest Discoveries of the $20^{\text {th }}$ Century. Tex Heart Inst J. 2002;29:164-71.

12. De Micheli A. El largo camino hacia la cardiología mexicana de hoy. Gac Méd Méx. 2004;140:347-52.

13. Gofman JW, Jones HB, Lindgren FT, Lyon TP, Elliot HA, Strisower B. Blood lipids and human atherosclerosis. Circulation. 1950;2:161-78.

14. Levine SA y Lown B. "Armchair" treatment of acute coronary thrombosis. J Am Med Assoc. 1952;148(16):1365-9.

15. Morris JN, Heady JA, Raffle PA, Roberts CG, Parks JW. Coronary heart-disease and physical activity of work. Lancet. 1953;265(6795):1053-7.

16. Pashkow $F$. Issues in contemporary cardiac rehabilitation: a historical perspective. J Am Coll Card. 1993;21:822-34

17. Mampuya W. Cardiac rehabilitation past, present and future: an overview. Cardiovasc Diag Ther. 2012;2:38-49.

18. Certo C. History of cardiac rehabilitation. Physical Therapy. 1985;65:1793-95.

19. Romero T. La rehabilitación cardíaca como punto de partida en la prevención secundaria de la enfermedad coronaria. Rev. méd. Chile. 2000;128:923-34.

20. Ruiz L, Devesa I. La medicina de rehabilitación: El ejercicio de la medicina en la segunda mitad del siglo XX. Tercera parte. Siglo XXI Editores;2005.

21. Pimentel Mercado E. Historia de la rehabilitación cardiaca. Centro Médico Nacional $2^{\circ}$ de noviembre. ISSSTE. Comunicación personal.

22. Férez Santander MS. Historia del Instituto Nacional de Cardiología y su influencia en la Medicina Mexicana. Comarketing Editorial; 2008.

23. Mehta NJ, Khan IA. Cardiology's 10 Greatest Discoveries of the $20^{\text {th }}$ Century. Tex Heart Inst J. 2002;29(3):164-71.

24. Reed H, Guazz M, Niebauer J. Cardiac rehabilitation in Europe. Progress in cardiovascular diseases. 2014;56:551-6.

25. Froelicher V, Jensen D, Atwood JE, McKirnan MD, Gerber K, Slutsky R, et al. Cardiac rehabilitation: evidence for improvement in myocardial perfusion and function. Arch Phys Med Rehabil. 1980;61(11):517-22.

26. Entrevista a Marcela Pérez Mancilla, fisioterapeuta del Servicio de Rehabilitación cardiaca del Instituto Nacional de Cardiología, 2016. Comunicación personal.

27. Oldridge N, Furlong W, Feeny D, Torrance G, Guyatt G, Crowe J, et al. Economic evaluation of cardiac rehabilitation soon after acute myocardial infarction. Am J Cardiol. 1993;72:154-61.

28. González M, Fernández de la Vega P, Ruiz Meza J, Leyva M, Ortiz E. Principales hospitales con servicios o programas de $\mathrm{RHC}$ en la Ciudad de México. Comunicaciones libres del XVIII Congreso Nacional de Cardiología de la Sociedad Mexicana de Cardiología. Archivos del Instituto Nacional de Cardiología; 1993. Abstract 518.

29. Oficio Secretaría de Marina. Comunicación personal, 1996.

30. Cantero Colín R. Historia de la rehabilitación cardiaca. Servicio de rehabilitación cardiaca. Centro Médico Nacional Siglo XXI. IMSS. Comunicación personal. 2018

31. Heberden W: Some account of a disorder of the breast. Medical transitions. Royal College of Physiscians, 1772. 